Advanced Computing: An International Journal ( ACIJ ), Vol.3, No.4, July 2012

\title{
STUDY AND IMPLEMENTATION OF ADVANCED NEUROERGONOMIC TECHNIQUES
}

\author{
Dr.B.F.Momin ${ }^{1}$ and Mrs. Mamata S.Kalas ${ }^{2}$ \\ ${ }^{1}$ Department of Computer Engineering, Walchand College of Engineering, Sangli, \\ MAHARASHTRA, INDIA \\ bfmomin@yahoo. com \\ ${ }^{2}$ Department of Information Technology, KIT'S College of Engineering, Kolhapur, \\ MAHARASHTRA, INDIA \\ mam2kalas2arediff.com
}

\begin{abstract}
Research in the area of neuroergonomics has blossomed in recent years with the emergence of noninvasive techniques for monitoring human brain function that can be used to study various aspects of human behavior in relation to technology and work, including mental workload, visual attention, working memory, motor control, human-automation interaction, and adaptive automation. Consequently, this interdisciplinary field is concerned with investigations of the neural bases of human perception, cognition, and performance in relation to systems and technologies in the real world -- for example, in the use of computers and various other machines at home or in the workplace, and in operating vehicles such as aircraft, cars, trains, and ships. We will look at recent trends in functional magnetic resonance imaging (fMRI), with a special focus on the questions that have been addressed. This focus is particularly important for functional neuroimaging, whose contributions will be measured by the depth of the questions asked. The ever-increasing understanding of the brain and behavior at work in the real world, the development of theoretical underpinnings, and the relentless spread of facilitative technology in the West and abroad are inexorably broadening the substrates for this interdisciplinary area of research and practice. Neuroergonomics blends neuroscience and ergonomics to the mutual benefit of both fields, and extends the study of brain structure and function beyond the contrived laboratory settings often used in neuropsychological, psychophysical, cognitive science, and other neurosciencerelated fields.
\end{abstract}

Neuroergonomics is providing rich observations of the brain and behavior at work, at home, in transportation, and in other everyday environments in human operators who see, hear, feel, attend, remember, decide, plan, act, move, or manipulate objects among other people and technology in diverse, real-world settings. The neuroergonomics approach is allowing researchers to ask different questions and develop new explanatory frameworks about humans at work in the real world and in relation to modern automated systems and machines, drawing from principles of neuropsychology, psychophysics, neurophysiology, and anatomy at neuronal and systems levels. The neuroergonomics approach allows researchers to ask different questions and develop new explanatory frameworks about humans at work in the real world and in relation to modern automated systems and machines. Better understanding of brain function can, for example, provide important guidelines and constraints for theories of information presentation and task design, optimization of alerting and warning signals, development of neural prostheses, and the design of robots. As an interdisciplinary endeavor, neuroergonomics will continue to benefit from and grow alongside developments in neuroscience, psychology,

DOI : $10.5121 /$ acij.2012.3402 
Advanced Computing: An International Journal ( ACIJ ), Vol.3, No.4, July 2012

\section{KEYWORDS}

Functional magnetic resonance imaging (Fmri), neuroergonomics, neuroscience, ergonomics

\section{INTRODUCTION}

In this, the 21 st century, human-brain mapping celebrates 21 years of cognitive activation studies. This review looks at imaging neuroscience and key ideas it has pursued; some ideas portend exciting developments, and others have failed gloriously. In terms of achievements, there is much to celebrate, in the sense that it is difficult to imagine modern neuroscience without brain imaging. We will look at recent advances from the perspectives of functional segregation and integration in the brain, paying special attention to approaches that deal with the distributed and integrated nature of neuronal processing and the questions they address.

Neuroimaging is now the predominant technique in behavioral and cognitive neuroscience. we can now characterize the integration of different brain areas in terms of functional and effective connectivity . Neuroergonomics is the application of neuroscience to ergonomics. Traditional ergonomic studies have relied largely on psychological explanations of issues of human factors, such as safety, response time, and repetitive stress injuries. Neuroergonomics, by contrast, relies on biological explanations.To meet these goals, neuroergonomics combines two disciplines--neuroscience, the study of brain function, and human factors, the study of how to match technology with the capabilities and limitations of people so they can work effectively and safely. The goal of merging these two fields is to use the startling discoveries of human brain and physiological functioning both to inform the design of technologies in the workplace and home, and to provide new training methods that enhance performance, expand capabilities, and optimize the fit between people and technology.

\section{RELATED WORK}

Neuroimaging methods will be used more effectively if the background in the information processing and cognitive engineering approaches to Ergonomics are kept in mind when trying to integrate these new methods and ideas from brain research[1]. According to [7], an imminent challenge in neuroergonomics will be to disseminate and advance new methods for measuring human performance and physiology in natural and naturalistic settings. It is likely that functional magnetic resonance imaging (fMRI) methods will be further applied to study brain activity in tasks that simulate instrumental activities of daily living, within the constraints of the scanner environment. The ability to image brain activity during complex, dynamic behavior such as driving and navigation tasks will enhance our understanding of the neural correlates of complex behaviors and the performances of neurologically normal and impaired individuals in the real world. Further use of fMRI paradigms involving naturalistic behaviors will depend on improved software design and analytic approaches, such as independent component analysis (ICA) to decompose and understand the complex data sets. This exciting future also depends on advances in brain imaging hardware, fMRI experimental design and data analyses, ever-better virtual reality (VR) environments, and stronger evidence to determine the extent to which tasks in these environments are valid surrogates for tasks in the real world.

The authors describe research and applications in prominent areas of neuroergonomics, Because human factors/ergonomics examines behavior and mind at work, it should include the study of brain mechanisms underlying human performance. Neuroergonomic studies are reviewed in four areas: workload and vigilance, adaptive automation, neuroengineering, and 
Advanced Computing: An International Journal ( ACIJ ), Vol.3, No.4, July 2012

molecular genetics and individual differences. Neuroimaging studies have helped identify the components of mental workload, workload assessment in complex tasks, and resource depletion in vigilance. Furthermore, real-time neurocognitive assessment of workload can trigger adaptive automation. Neural measures can also drive brain-computer interfaces to provide disabled users new communication channels. Finally, variants of particular genes can be associated with individual differences in specific cognitive functions. Neuroergonomics shows that considering what makes work possible - the human brain - can enrich understanding of the use of technology by humans and can inform technological design. Applications of neuroergonomics include the assessment of operator workload and vigilance, implementation of real-time adaptive automation, neuroengineering for people with disabilities, and design of selection and training methods.

According to [31], individualized adaptive training platforms offer promise in meeting the increasing demands on people for information acquisition and mastery of new skills. Learning is most efficient when working memory resources are managed effectively. Efficient instruction presents training materials at a pace and in a format that keeps a learner engaged and motivated without overloading his or her limited working memory resources. Adaptive computer-based training has typically been driven by performance metrics. Consistent with the neuroergonomic approach, however, measures of brain function may provide additional or complementary information to behavioral measures for use in adaptive training. Neuroergonomic methods of classifying a learner's mental workload (defined here as working memory processing load) show promise for driving real-time adaptive training platforms. Crucial to their implementation, however, is the ability to identify the learner's workload when new tasks or more difficult levels of a familiar task are encountered. The current study examined the accuracy of an artificial neural network (ANN) based classification algorithm based solely on electroencephalographic (EEG) activity obtained from novice performers. Specifically, we sought to determine the extent to which an ANN trained to identify difficulty levels in one task could classify workload on a different task when the operator has little to no experience performing either task. Crosstask classifiers capable of determining a learner's current workload and cognitive-emotional response during training are essential for implementing adaptive training. EEG as an index of workload Learning depends heavily on working memory, which has well documented limitations.

Furthermore, working memory capacity has been shown to vary across individuals and serves as a strong predictor of problem solving ability, retention, reading comprehension, the ability to integrate information, the ability to inhibit irrelevant information and a number of other processes essential to learning. Several investigations indicate that classification algorithms such as ANNs show great promise for effective real-time workload classification. Artificial neural network parameters MATLAB and the Neural Network Toolbox extension were used to simulate the ANNs for this experiment. ANNs were randomly initialized multi-layer perceptrons trained using traditional feedforward testing and back propagation techniques with scaled conjugate gradients for increased speed. For all ANNs, the number of hidden layer nodes was kept consistent at 20. The EEG inputs described above were target-coded using a one-of-N dummy variable scheme (i.e., low and high difficulty inputs' target outputs were ' 1,0 ' and ' 0 , 1' respectively). Therefore, each ANN had 40, 45, or 50 (2, 1, or no rejected channels, respectively) input nodes, 20 hidden nodes in one hidden layer, and 2 output nodes. The decision to use 2 output nodes instead of 1 scalar output for the ANN was based on peculiar classification behavior (e.g., extremely inconsistent training due to early divergence) observed during initial analyses. Each neural network was trained using $50 \%$ of data for training and the 
Advanced Computing: An International Journal ( ACIJ ), Vol.3, No.4, July 2012

remaining 50\% for validation. Data was divided randomly between training and validation sets. Training continued until neural network error on the training set reached a predefined minimum limit or error on the validation set began to diverge so as to avoid overtraining.

In [21],Neuroergonomics integrates knowledge of brain function with measurements of behavior that are acquired "at work" to develop methods that can enhance performance .This paper reviews recent experiments where the work at hand involves observing motor behavior performed by other people and using this information in forming judgments, decisions, or actions. Body movement comes in many forms, from changes of posture to the grasping or manipulation of a tool. This review considers how some of these complex and varied behaviors are decoded or interpreted in the brain, with a long range goal of improving work environments requiring judgments about another person's actions or intention. These work environments are plentiful and increasing. The worker is faced with maintaining high classification accuracy in the face of severe demands such as high throughput, stress, and potential physical threat. Almost every security force in the world is faced with making fast and accurate decisions about the intentions of human agents. Familiar examples include border security (passport control), standoff procedures (checkpoints), high throughput screening (TSA) and video surveillance (security cameras). In all these examples, impending actions are uncertain, and situated in rich and changing contexts and shaped by an enormous range of cultural influences. By combining real-time fMRI monitoring and neuro feedback during training, future research may be able to monitor the effects of training parameters on changes in the interaction of brain systems, perhaps improving the ability to adopt the most effective learning strategies. Ultimately these techniques may also help characterize how training-induced changes in functional connectivity are related to retention and transfer of learned skills to real-world environments such as driving, sport, or neurorehabilitation. For example, successful transfer of training may require both context-specific functional plasticity as well as functional plasticity of resting networks. In sum, the current study gives us novel insight into the mechanisms underlying the effectiveness of VP training while also demonstrating new possibilities for using functional neuroimaging to examine the brain at work. The neuroergonomic approach integrates naturalistic task demands with methodologies and insight from cognitive neuroscience. The ultimate goal is to enhance performance through changes in training or the modification of information processing in real time using neural signatures. In this review, future work is based on (1) development of sensor systems and analysis streams for brain-computer systems to augment or accelerate an observer's decision making; (2) creation of training tools, including immersion, that shape the observer's performance based on brain responses; and (3) the evolution of monitors, workstations and work environments to adapt information to features that the observer depends on.

Molecular genetic studies of individual differences provide a final example of neuroergonomics research. These studies have capitalized on the recent decoding of the human genome and on neuroimaging studies that have linked cognitive functions to the activation of specific cortical networks . Gene expression can influence the efficiency of these networks - for example, through modulation of neurotransmitters innervating a particular network. Some (but not all) genes come in different forms (alleles), with one of the two alleles in a paired DNA strand being inherited from each parent. A given person may have none, one, or two alleles in a specified location within the gene. One then can examine the functional consequence, if any, of such allelic variation. Studies using this approach have shown that individual differences in cognitive functioning can be linked to variations in specific genes . 
Advanced Computing: An International Journal ( ACIJ ), Vol.3, No.4, July 2012

The future of neuroergonomics seems assured because of its initial successes and because of the ever increasing understanding of the brain and behavior at work in the real world. Neuroergonomics blends neuroscience and ergonomics to the mutual benefit of both fields and extends the study of brain structure and function beyond the basic tasks used in cognitive psychology and neuroscience. Neuroergonomics can provide rich observations of the brain and behavior at work, at home, in transportation, and in other everyday environments involving human operatorsIn [19].Non-invasive optical imaging tools, such as near-infrared spectroscopy (NIRS), assess neuronal activity that occurs directly after stimulus presentation or in preparation for responses and hemodynamic changes In [19] emphasis is made about neuroergonomic assessment should be used in the context of current and future task demands on the operator. Using only physiological measures during complex system operation could result in inappropriate AA. For example, landing an aircraft produces signs of increased workload, which are expected and in the context of landing. AA should not be implemented unless even higher workload is detected. An adaptive system manager would determine the intervention that reduces workload in the current task situation. In complex job situations, a number of mitigations would be available, but only the ones that are appropriate at a given time would be selected. Whereas current research has had the goal of detecting existing mental overload, future research should focus on detecting when an operator is becoming overloaded. Using this information, people could implement interventions to prevent overload. This would have the effect of further reducing errors and improving job success. States other than high mental workload-fatigue, vigilance, and inattention - should also be investigated.

In [5], the relatively new approach of using noninvasive brain stimulation technologies presented does not preclude the further investigation of applied science questions. Rather, brain stimulation for the sake of cognitive enhancement in "normal" subjects expands the domain, applicability, development, and understanding of traditional stimulation research. As noninvasive brain stimulation research and technology continues to evolve and researchers answer fundamental questions, the neuroergonomics community should strive to apply brain stimulation techniques in more complex domains. With a more developed skill set and understanding, scientists and engineers will then be in a better position to transition brain stimulation technologies into operational communities. Because constant stimulation is less than ideal, it is important to develop methodologies for smart activation of stimulation devices only when needed for the maintenance of optimal operator performance. One possibility resides in utilizing physiological parameters as a feedback mechanism for a closed-loop brain stimulation system. However, these physiological metrics must have a robust correlation with the cognitive skill/task performance in order to be effective. While a considerable amount of research remains to be conducted, noninvasive brain stimulation provides an exciting potential method for positively augmenting the human operator to improve efficiency, reduce training, and enhance a variety of cognitive skills that should ultimately improve performance at work.

According to [5,6], Naturalistic paradigms such as movie watching or simulated driving that mimic closely real-world complex activities are becoming more widely used in functional magnetic resonance imaging (fMRI) studies both because of their ability to robustly stimulate brain connectivity and the availability of analysis methods which are able to capitalize on connectivity within and among intrinsic brain networks identified both during a task and in resting fMRI data.

Neuroergonomics merges neuroscience and ergonomics for the study of brain and behavior in natural and naturalistic settings. Together with the rapid development of neuroergonomics concepts, echnologies, and related data, there is an urgent need to develop computational 
Advanced Computing: An International Journal ( ACIJ ), Vol.3, No.4, July 2012

models of neuroergonomics that can help integrate and interpret empirical findings and make predictions for scientific research and practical application. The article[37] discusses the relationship between computational neuroscience and computational neuroergonomics, and describes a queuing network based computational neuroergonomic architecture and its applications. These discussions illustrate the mission and challenges of computational neuroergonomics and future research needs[1].

Computational modeling can help researchers and practitioners organize and summarize empirical data, provide unique perspectives to examine and interpret the data, guide and generate hypotheses for new experiments to obtain data in a systematic fashion, and make extrapolations and predictions to situations in which empirical data collection is not feasible or undesirable. Scientifically, computational modeling help advance the understanding of the brain and behavior; practically, it may help guide system design by comparing different design alternatives and predicting human behavior in operational contexts that cannot be easily covered by experimental or simulation studies[1].

Neuroergonomic applications of the QN architecture describes several illustrative examples of neuroergonomic applications of the QN architecture, focusing on multitask modeling. Modeling of multitask performance is one of the major challenges of cognitive and ergonomic modeling, since performing multiple tasks at the same time is common in daily life and has significant safety and design implications; for example, drivers can steer a car and at the same time talk with friends in the car, and telephone operators can answer customer phone calls and type textual information into a computer.

From the perspective of neuroergonomic applications, it is instructive to use the four applications of neuroergonomics described to illustrate the challenges of the QN architecture. This illustration pertains to computational neuroergonomics in general as well. In [3] authors described four applications of neuroergonomics, namely the assessment of operator workload and vigilance, implementation of real-time adaptive automation, neuroengineering for people with disabilities, and design of selection and training methods. In this regard, the QN has successfully modeled a variety of subjective and ERP-based workload data[23] and an adaptive automation system has been developed for driver interface design[24] while no other computational neuroscience or neuroergonomic model has tackled these two issues. However, the QN has not been applied to neuroengineering for people with disabilities, nor to the design of selection and training methods. Conceptually, it is feasible for the QN to explore these and related issues. For example, genetics and individual differences as well as people with disabilities can be represented in the QN as different service routes, different service rates at corresponding servers, or other parameters and values. Testing the various modeling methods while absorbing and predicting related findings, however, remains a challenge ahead. Meeting this challenge requires a systematic and disciplined approach[1].

\section{PROPOSED WORK}

The objective of present research is

- To monitor the effects of training parameters on changes in the interaction of brain systems, by combining real-time fMRI monitoring and neurofeedback during training, perhaps improving the ability to adopt the most effective learning strategies.

- Current research has had the goal of detecting existing mental overload, future research focus on detecting when an operator is becoming overloaded. 
Advanced Computing: An International Journal ( ACIJ ), Vol.3, No.4, July 2012

- To model the multitask performance, which is one of the major challenges of cognitive and ergonomic modeling, since performing multiple tasks at the same time is common in daily life and has significant safety and design implications;

- To develop methodologies for smart activation of stimulation devices only when needed for the maintenance of optimal operator performance.

- To devise noninvasive brain stimulation which provides an exciting potential method for positively augmenting the human operator to improve efficiency, reduce training, and enhance a variety of cognitive skills that should ultimately improve performance at work.

- To design more sophisticated techniques, with an integration of EEG techniques to measure mental workload in human operators in monitoring and adaptive aiding systems.

- To model Queuing Network architecture for genetics and individual differences as well as people with disabilities.

\section{METHODOLOGY.}

This proposed research work is related to the processing of the sample datasets of MR images of brain. So preprocessing steps are performed on these images. Functional MRI data acquisition done Functional images are acquired with gradient-recalled echoplanar imaging.. fMRI analyses are carried out using the FSL software package and MATLAB. To examine functional connectivity in select brain systems, or the extent to which distributed networks activated cohesively in space and time, we follow a standard pipeline that is used for functional connectivity analysis. This includes typical functional image processing steps such a brain extraction, motion correction, spatial smoothing.

Statistical analysis of brain-behavior relationships

For examination of brain-behavior relationships, we hypothesize that functional connectivity in the DMN (Default Node Networks) would serve as a significant source of variance in the association between aerobic fitness and cognition. We are going to justify the causal directions of the model such that it is unlikely functional connectivity will cause aerobic fitness; similarly it is unlikely in this cross-sectional analysis that cognition would cause functional connectivity. While causal predictions in correlational data deserve caution, we believe the proposed model provides the best theoretical framework for the associations between fitness, cognition, and functional connectivity. In the model the paths between the independent variable (IV) (fitness) and the mediator variable (M) (functional connectivity) and $\mathrm{M}$ to the dependent variable (DV) (cognition) are to be represented with the coefficients $a$ and $b$ respectively. Here a represents the coefficient of the relationship between the IV and M, and $b$ represents DV; together $a \times b$ represents the indirect effect of functional connectivity on cognition. A third coefficient, $\mathrm{c}$, then represents the coefficient of the relationship between the IV and DV, or the direct effect of fitness on cognition.

\section{b) Data base and sources:}

1. Study and research work requires data which contains MRI images obtained from Slicer User Orientation[] Slicer is an open-source software platform for the analysis and visualization of medical images and for research in image guided therapy. The platform provides functionality for segmentation, registration and three-dimensional visualization of multi-modal image data, as well as advanced image analysis algorithms for diffusion tensor imaging, functional magnetic resonance imaging and image-guided therapy. Standard image file formats are supported, and the 
Advanced Computing: An International Journal ( ACIJ ), Vol.3, No.4, July 2012

application integrates interface capabilities to biomedical research software and image informatics frameworks. This data will be used for the research work.

2. The MRI cognitive toolbox of FMRIB centre for clinical neurosciences used to get the interpretations. FEAT[www.fmrib.ox.ac.uk/fsl) - an advanced FMRI analysis tool with easy-touse yet powerful GUI for complete first- and higher-level FMRI analysis.

3. Functional MRI data analysis Initial data preprocessing utilizes SPM5 (Wellcome Department of Cognitive Neurology, London, UK) for slice acquisition correction, motion correction, coregistration, and spatial normalization. fMRI analyses were carried out using the FSL software package and MATLAB.

\section{c) Design Methodology:}

I. In the present problem, Neuroimaging techniques for providing Connectivity, and correlation of neural network activity in neuro imaging, Bayesian models, Gaussian mixture models for fMRI will be developed.

II. Major techniques and methods applied in network analysis of neuroimaging data as a way to examine possible interaction patterns between brain regions, Structural equation modeling (SEM) and dynamic Bayesian networks (DBN) are both statistical causal modeling methods widely used Pure computational symbolic models lack mathematical representations of their overall structures at the macro architectural level. So, the processing of data mainly depends on the mathematical equations or models needed to capture the whole range of concurrent processing activities, affective states, and neural mechanisms.

III. We develop model the multitask performance which is one of the major challenges of cognitive and ergonomic modeling, since performing multiple tasks at the same time is common in daily life and has significant safety and design implications.

IV. Developing better models that complement and exploit the richness of these data.

V. Developing New mathematical models and devise novel algorithms for implementation of functional connectivity between brain regions.

VI. Analysis and simulation of these models and algorithms are developed.

\section{EXPERIMENTAL WORK}

The proposed research work requires both theoretical and experimental work. Theoretical work will be based on equations solving, derivation of the formulae and building algorithms for computation of the complicated mathematical models. Data is collected from various sources like different open source softwares(Slicer), as well as $\mathrm{m}$ fMRI preprocessing is to be carried using FSL 4.1.2 (FMRIB's Software Library, www.fmrib.ox.ac.uk/fsl). The following prestatistics processing is to be applied: rigid body motion correction using MCFLIRT (Jenkinson et al., 2002), removal of non-brain structures using BET (Smith, 2002), spatial smoothing using a Gaussian kernel, grand-mean intensity normalization of the entire 4D dataset by a single multiplicative factor, and temporal filtering with a high pass frequency.

General linear model analysis for initial seed selection Regression-based analysis of fMRI data is to be carried out using FEAT (FMRI Expert Analysis Tool, http://www.fmrib. ox.ac.uk/analysis/research/feat/) Version 5.98, part of FSL. At the individual level, a separate individual level analysis is going to done for each of the passive viewing scans. The 
Advanced Computing: An International Journal ( ACIJ ), Vol.3, No.4, July 2012

hemodynamic response to each block is convolved with a double-gamma HRF function. We choose to use a double-gamma HRF function since preliminary analysis of the checker stimulation task showed that the convolution of the double-gamma function had a better fit to the data than the gamma function. The double-gamma models an initial overshoot, followed by a post-HRF-undershoot, and this showed to be the best fit to the signal for both young and elderly adults. Furthermore, the same high-pass temporal filtering that was applied to the data was applied to the GLM for the best possible match between the model and data. For each task there was a linear contrast that tested for the comparison of activation during rest greater than visual processing blocks (rest $>$ task). The three statistical maps that resulted (rest $>$ task for each of the three passive viewing tasks) are then combined into a higher-level fixed-effects analysis, which generated a statistical map representing where activation is greatest for rest $>$ visual processing across all three fMRI scans, within a subject. Statistical maps from the fixedeffects analysis were then forwarded to a higher-level mixed-effects analysis to find areas across participants that are active during rest than visual processing. Higher-level mixed-effects analyses are to be carried out using FLAME [29].

To ensure regional differences in gray matter volume did not confound our analyses, gray matter partial volume 3D images are added as voxel-wise covariates in the functional imaging analysis[30]7; implemented as part as FMRI Expert Analysis Tool Version 5.98, part of FSL 4.1.2, FMRIB's Software Library,(www.fmrib.ox.ac.uk/fsl)

New stimulation models are defined and developed for different neuroergonomic activities for assessing the workload of the brain in resting state, at work and providing connectivity, correlations, and modeling computational model. We will design and implement new algorithms to meet the challenges posed by neuroimaging techniques (Registration algorithms, novel approach to regression algorithms, and Independent Component Analysis (ICA) Algorithm. And computing the efficiency of the designed methods and algorithms compared with existing ones for behavioural, functional connectivity and statistical inference based analysis. Experimental work will be focused on implementation of new algorithms and models for processing the MRI .

\section{CONCLUSIONS}

As with any merger of previously unrelated scientific disciplines, the usefulness of combining cognitive neuroscience and cognitive ergonomics to create the new field of 'neuroergonomics' depends on the significance of the research subjects that could not be addressed if the two fields remained separated. Situations characterized by a scarcity of overt operator behavior and performance represent one driving force for combining the two fields. In those situations, neurobiological indices of cognitive processes and capacities may emerge as the main, or even exclusive, source of information about the operator's cognitive status and activities. This information is needed, for example, to support adaptive aiding and dynamic function allocation in the context of human-automation cooperation. The utilization of research approaches and measurements more typical of cognitive neuroscience will benefit the field of cognitive ergonomics to the extent that multidimensional psychological constructs (such as situation awareness and mental workload) are reduced to their underlying cognitive operations which are more isomorphic with activity changes in brain regions, circuits and distributed neuronal systems. The transformation of these constructs and paradigms will, in turn, enable cognitive ergonomics to contribute to progress in the field of cognitive neuroscience. 
Advanced Computing: An International Journal ( ACIJ ), Vol.3, No.4, July 2012

\section{REFERENCES}

[1] Michael I. Posne 'Expanding horizons in ergonomics research' NeuroImage 59 (2012) pg 149-153.

[2] Parasuraman, R., 2011. Neuroergonomics: brain, cognition and performance at work.Curr. Dir. Psychol.Sci.20,181-186.

[3]. Gleick, J., 2011. The Information: A History, a Theory, a Flood. Pantheon, New York.

[4] Crottaz-Herbette, S., Menon, V., 2006. Where and when the anterior cingulate cortex modulates attentional response: combined fMRI and ERP evidence. J. Cogn.Neurosci. 18, 766-780.

[5] V.D. Calhoun G.D. Pearlson, A selective review of simulated driving studies: combining naturalistic and hybrid paradigms, analysis approaches, and future direction, NeuroImage 59 (2012) 25-35

[6] Voss, M.W., 2012. Effects of training strategies implemented in a complex videogame on functional connectivity of attentional networks. Neuroimage 59, 138-148.

[7] Matthew Rizzo and Raja Parasuraman Future Prospects for Neuroergonomics

[8] Clark, A. (2003). Natural born cyborgs: Minds, technologies, and the future of human intelligence. NewYork: Oxford University Press.

[9] De Pontbriand, R. (2005). Neuro-ergonomics support from bio- and nano-technologies, In Proceedings ofthe Human Computer Interaction International Conference. Las Vegas, NV: HCI International.

[10]Gevins, A., Leong, H., Du, R., Smith, M., Le, J., DuRousseau, D., Zhang, J., \& Libove, J. (1995).Towards measurement of brain function in operational environments. Biological Psychology, 40,169-186.

[11]Hancock, P.A., \& Szalma, J.L. (2003). The future of neuroergonomics. Theoretical Issues in ErgonomicsScience, 4, 238-249.

[12]Parasuraman, R. (2003). Neuroergonomics: Research and practice. Theoretical Issues in ErgonomicsScience, 1-2, 5-20.

[13]St. John, M., Kobus, D. A., Morrison, J. G., \& Schmorrow, D. (2004). Overview of the DARPA augmented cognition technical integration experiment. International Journal of HumanComputerInteraction, 17, 131-149.

[14]Vertegaal, R. (2002). Designing attentive user interfaces. In Proceedings of the Symposium on EyeTracking Research and Applications. (pp. 23-30). New Orleans, Louisiana: SIGCHI.

[15] Raja Parasuraman, George Mason University, Fairfax, Virginia, and Glenn F. Wilson,Physiometrex, Dayton, Ohio Putting the Brain to Work: Neuroergonomics Past, Present, and Future, HUMAN FACTORS, Vol. 50, No. 3, June 2008, pp. 468-474. DOI 10.1518/001872008X288349. Copyright (C) 2008, Human Factors and Ergonomics Society.

[16] Hasan Ayaz ,Patricia A. Shewokis,,Scott Bunce, Kurtulus Izzetoglu, Ben Willems, Banu Onaral Optical brain monitoring for operator training and mental workload assessment NeuroImage 59 (2012) 83-93

[17] Kelly, A., Garavan, H., 2005. Human functional neuroimaging of brain changes associated with practice. Cerebral Cortex 15, 1089.

[18] Parasuraman, 2003; Parasuraman \& Rizzo, 2007,Neuroergonomics is the study of brain and behavior at work

[19] R. Andy McKinley, Nathaniel Bridges, Craig M. Walters, Jeremy Nelson Modulating the brain at work using noninvasive transcranial stimulation ,5 August 2011 
Advanced Computing: An International Journal ( ACIJ ), Vol.3, No.4, July 2012

[20] Ayaz, H., Shewokis, P.A., Bunce, S., Izzetoglu, K.,Willems, B., Onaral, B., 2012. Optical brain monitoring for operator training and mental workload assessment. Neuroimage 59,36-47 .

[21] Clark, V.P., Coffman, B.A., Mayer, A.R., Weisend, M.P., Lane, T.D.R., Calhoun, V.,Raybourn, E.M., Garcia, C.M., Wassermann, E.M., 2012. TDCS guided using fMRI significantly accelerates learning to identify concealed objects. Neuroimage 59,117-128

[22] Grafton, D.T., Tipper, C.M., 2012. Decoding intention: A neuroergonomic perspective.Neuroimage $59,14-24$

[23] Parasuraman, R., 2011. Neuroergonomics: brain, cognition and performance at work.Curr. Dir. Psychol. Sci. 20, 181-186.

[24] Parasuraman, R., Jiang, Y., 2012. Individual differences in cognition, affect, and performance: Behavioral, neuroimaging, and molecular genetic approaches. Neuroimage

[25] Wu, C., Liu, Y., 2007. Queueing network modeling of driver performance and workload. IEEE Trans. Intell. Transport. Syst. 8, 528-537

[26] Wu, C., Liu, Y., 2008b. Queueing network modeling of transcription typing. ACM Trans.Comput. Hum. Interact. 15, 1-45.

[27] Matthew Rizzo and Raja Parasuraman,Future Prospects for Neuroergonomics Theoretical Issues in ErgonomicsScience, 2007

[28] Marcus E. Raichle A Paradigm Shift in Functional Brain Imaging The Journal of Neuroscience, October 14, 2009 • 29(41):12729-12734・12729

[29] Beckmann, C. F., Jerome, G. J., \& Smith, S. M. (2003). General multilevel linear modeling for group analysis in fMRI. Neuroimage, 20, 1052-1063.

[30] Oakes, T. R., Fox, A. S., Johnstone, T., Chung, M. K., Kalin, N., \& Davidson, R. J. (2007). Integrating VBM into the general linear model with voxelwise anatomical covariates. NeuroImage, 34, 500-508.

[31] Carryl L. Baldwin , B.N. Penaranda, Adaptive training using an artificial neural network and EEG metrics for within- and cross-task workload classification, NeuroImage, 59 (2012) 48-56.

\section{Authors:}

Dr. Bashirahamad F. Momin is working as Associate Professor \& Head, Dept. of Computer Science \& Engineering, Walchand College of Engineering Sangli, Maharashtra State India. He received the B.E. and M.E. degree in Computer Science \& Engineering from Shivaji University, Kolhapur, India in 1990 and 2001 respectively. In February 2008, he had completed his Ph.D. in Computer Science and Engineering from Jadavpur University, Kolkata. He is recognized Ph.D. guide in Computer Science \& Engineering at Shivaji University, Kolhapur. His research interest includes pattern recognition \& its applications, data mining and soft computing techniques, systems implementation on the state of art technology. He was a Principal Investigator of R \& D Project titled "Data Mining for Very Large Databases" funded under RPS, AICTE, New Delhi, India. He had delivered a invited talk in Korea University, Korea and Younsea University, Korea. He had worked as "Sabbatical Professor" at Infosys Technologies Ltd., Pune. He is a Life Member of "Advanced Computing and Communication Society", Bangalore INDIA. He was a student member of IEEE, IEEE Computer Society. He was a member of International Unit of Pattern Recognition and Artificial Intelligence (IUPRAI) USA. 
Mrs. Mamata S. Kalas, Having been graduate from University Of Mysore, in 1993, From B.I.E.T,Davangere, started her professional carrier there itself. In 1995, came to kolhapur, after her marriage and since then she has worked as lecturer at D.Y.Patil's college of Engg.,Bharati Vidyapeeth's College Of Engg.,Kolhapur.She is M.Tech(CST) Graduate and her dissertation work is based on image segmentation using parametric distributional clustering. She has been awarded with M.TECH (CST) from Shivaji University, Kolhapur of Maharashtra in June 2009. Recently she has been registered for $\mathrm{Ph} . \mathrm{D}$ in computer science and Engineering at walchand College of Engineering, Reseach center, Shivaji University, under the guidance of Dr.B.F.Momin. She is currently working as an Assistant Professor at KIT'S College of Engg, Kolhapur.She is in her credit, 16 Years of teaching experience, Two Papers presented for international conferences, three papers presented for national conferences, seven papers published in international journals. Her areas of interest are pattern recognition and artificial intelligence, computer architecture, system programming.

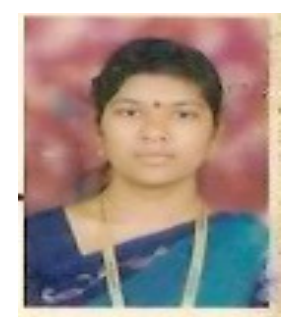

